\title{
Density Functional Study of Bulk and Surface Properties of Rhodium Hydride
}

\author{
G. Sudhapriyanga ${ }^{a}$, A.T. AsvinimeenaAtci ${ }^{a}$, R. Rajeswarapalanichamy ${ }^{a} *$ \\ AND K. IYAKUTTI ${ }^{b}$ \\ ${ }^{a}$ N.M.S.S. Vellaichamy Nadar College, Madurai, Tamil nadu-625019, India \\ ${ }^{b}$ Department of Physics and Nanotechnology, SRM University, Chennai, Tamil nadu-603203, India
}

(Received May 9, 2012; in final form September 24, 2013)

\begin{abstract}
The electronic properties and structural phase transition of bulk rhodium hydride are analyzed using density functional theory calculations with the generalized gradient approximations. The sequent phase transition is observed in bulk rhodium hydride. The predicted new high pressure phase of rhodium hydride is hexagonal NiAs type. The atomic geometry, adsorption energy, and binding energy of the Rh (111) surface are computed. The calculated surface energy for Rh (111) surface is $1.06349 \mathrm{eV}$ and the maximum adsorption energy is obtained in $6 \times 1$ phase as $2.8617 \mathrm{eV}$. The relaxed geometries show that hydrogen has a strong influence on the interlayer distance.
\end{abstract}

DOI: $10.12693 /$ APhysPolA.125.29

PACS 31.15.A-, 61.50.Ks, 62.20.-x, 68.35.Md

\section{Introduction}

The $4 d$ transition metal hydrides attract renewed interest as a potential hydrogen source for portable fuel cells [1]. Rhodium has several interesting mechanical and structural properties. Irodova et al. [2] investigated the formation of $4 d$ transition metal hydrides experimentally using neutron diffraction method and they predicted rhodium hydride crystallized in $\mathrm{NaCl}$ structure. The structural and magnetic properties of $\mathrm{RhH}$ were analyzed using ab initio studies [3]. Bannikov et al. [4] investigated the electronic structure and magnetic properties of $\mathrm{RhH}_{x}$ $(x=0.25,1.0,1.33)$ based on full-potential linearized augmented plane wave generalized gradient approximation (FLAPW-GGA) calculations. Using the first principles calculation, Xin Cui et al. [5] reported the mechanical and magnetic properties for both $\mathrm{Rh}$ and $\mathrm{RhH}$ systems. Rhodium is an active catalyst in various reactions involving hydrogen interaction with several reagents [6-11]. Hence understanding the nature of hydrogen interaction with a rhodium surface is essentially important. The hydrogen adsorption on polycrystalline $\mathrm{Rh}$ $[7,12-14]$ and on rhodium single crystals [15-22] were reported. The experimental studies of hydrogen adsorption on Rh (110) [16-21] and Rh (111) [15-22] surfaces showed an interesting feature. The investigation of structural features of Rh (100) surface was performed by Eichler et al. [23], no other theoretical information has been reported yet on $R h$ (111) surface.

To the best of our knowledge, investigation of mechanical stability and structural phase transition of rhodium hydride under high pressure have not been reported yet.

*corresponding author; e-mail: rrpalanichamy@gmail.com
In this work, we have theoretically investigated the mechanical stability, structural phase transition, electronic properties of bulk rhodium hydride and the stability of rhodium thin slab (Rh (111) and Rh (100)) via surface energy calculation. We have also analyzed the adsorption of hydrogen on Rh (111) surface slab via periodic, self-consistent density-functional theory (DFT) calculations. The preferred adsorption sites, optimized geometries and surface binding energy all have been presented and analyzed.

\section{Computational details}

All the calculations in this study are based on density functional theory as implemented in the Vienna $a b i n i$ tio simulation package (VASP) code [24-28], using the projector augmented wave (PAW) method [29, 30] and the generalized gradient approximation (GGA) with the Perdew-Burke-Ernzerhof (PBE) [31] formulation was used to describe the exchange-correlation contributions. We have used a plane-wave basis set to expand the electronic wave-functions with the same plane-wave energy cutoff of $400 \mathrm{eV}$, which guarantees convergence of adsorption energies within $1 \mathrm{meV}$. The Brillouin zone integrations are performed on the Monkhorst-Pack $K$-point mesh [32] with a grid size of $12 \times 12 \times 12$ for structural optimization and the total energy calculation of bulk $\mathrm{RhH}$ $[3,5]$. Surfaces were modeled using periodic slabs, with five atomic layers and a vacuum thickness of $10.53 \AA$. Adsorption was allowed on only one of the two exposed surfaces, and the electrostatic potential was adjusted accordingly. Four different cells, i.e., $3 \times 1,4 \times 1,5 \times 1$, and $6 \times 1$, were considered for the adsorption of hydrogen on $\mathrm{Rh}(111)$ surface. The adsorbate and the atoms in the top three layers were allowed to relax while the atoms in the bottom two layers were fixed at the bulk truncated positions. Calculations were performed using 
$2 \times 2$ surface unit cells, with $9 \times 9 \times 1 k$-point grids . Based on the convergence of total energies, for Rh surfaces, a $9 \times 9 \times 1$ Monkhorst-Pack grid was employed for the (111) and (100) surfaces, respectively. For all of the relaxations, an energy difference of $10^{-4} \mathrm{eV}$ was set as the convergence criterion between successive ionic steps. When the maximum force acting on each atom of the slab dropped below $0.01 \mathrm{eV} / \AA$, structural relaxation calculations were stopped. The optimized lattice constants of bulk Rh was $a=3.80 \AA$. The atomic slabs are separated in the $z$-direction by vacuum regions. The vacuum space was set to $10.53 \AA$ to guarantee a sufficient separation between the periodic images.

\section{Results and discussion}

\subsection{The optimized crystal structures and formation energies}

Many of the transition metal monohydrides are stable in cubic-NaCl structure [33-40] or hexagonal structure at ambient pressure [35, 41] and it may undergo a structural phase transition under high pressure $[38,39]$. Hence, we have investigated the different cubic and hexagonal crystal structures for rhodium hydride such as $\mathrm{NaCl}$ (space group $F m \overline{3} m$ ), $\mathrm{CsCl}$ (space group $P m \overline{3} m$ ), ZB (space group $F \overline{4} 3 m$ ), WC (space group $P 6_{3} \bar{m} c$ ) and NiAs (space group $\mathrm{P}_{3} / \mathrm{mmc}$ ) structures. Since the formation energy of these structures is negative, it can be easily formed under pressure. Among these crystal structures, NaCl-RhH is the most stable structure.

The equilibrium lattice parameters are determined by minimising the total energy for different values of lattice constants [42]. The valence electron density (VED) is defined as the total number of valence electrons divided by volume per unit cell, which is an important factor for analyzing the super hard materials.

\section{TABLE I}

Calculated lattice parameters $a, \quad c(\AA)$, equilibrium volume $V_{0}\left(\AA^{3}\right)$, valence electron density VED (electrons $\left./ \AA^{3}\right)$, bulk modulus $B_{0}(\mathrm{GPa})$, and pressure derivative $B_{0}^{\prime}$ for the rhodium hydride for five different structures.

\begin{tabular}{|c|c|c|c|c|c|}
\hline & $\mathrm{NaCl}$ & $\mathrm{CsCl}$ & ZB & WC & $\mathrm{NiAs}$ \\
\hline$a$ & 3.8303 & 2.689 & 4.2116 & 3.371 & 2.844 \\
\hline$c$ & & & & 4.256 & 3.892 \\
\hline & $4.010[2]$ & & & & \\
\hline & $4.053[3]$ & & & & \\
\hline & $4.084[4]$ & & & & \\
\hline & $4.044[5]$ & & & & \\
\hline$V_{0}$ & 13.72 & 19.44 & 18.68 & 21.27 & 0.3595 \\
\hline$\rho$ & 0.728 & 0.5144 & 0.5353 & 0.4701 & 192 \\
\hline$B_{0}$ & $201[5]$ & 229 & 218 & 160 & 192 \\
\hline$B_{0}^{\prime}$ & 3.71 & 3.6 & 3.76 & 3.94 & $\begin{array}{l}3.42 \\
-3.402\end{array}$ \\
\hline $\begin{array}{l}\text { formation } \\
\text { energy }\end{array}$ & -4.7547 & -3.8331 & -4.5032 & -2.9617 & -3.402 \\
\hline
\end{tabular}

The equilibrium lattice parameters $a, c(\AA)$, volumes of unit cells $V_{0}\left(\AA^{3}\right)$, valence electron density
VED (electrons $/ \AA^{3}$ ), formation energy per unit cell $(\mathrm{eV})$, bulk modulus (GPa) and its pressure derivative along with the experimental [34] and other available theoretical values [20-22] for five different crystal structure of rhodium hydride are given in Table I. The calculated VEDs are 0.728 electrons $/ \AA^{3}$ for $\mathrm{NaCl}-\mathrm{RhH}$, 0.5144 electrons $/ \AA^{3}$ for CsCl-RhH, 0.5333 electrons $/ \AA^{3}$ for $\mathrm{ZB}-\mathrm{RhH}, 0.4701$ electrons $/ \AA^{3}$ for $\mathrm{WC}-\mathrm{RhH}, 0.3595$ electrons $/ \AA^{3}$ for NiAs-RhH. It is worth to note that the VED of $\mathrm{NaCl}-\mathrm{RhH}$ is comparable to 0.70 electrons $/ \AA^{3}$ for diamond [43]. Among the considered structure $\mathrm{NaCl}-$ $\mathrm{RhH}$ have the highest VED and it is found to be the hardest material. The primitive unit cells of different crystal structure of rhodium hydride are shown in Fig. 1.

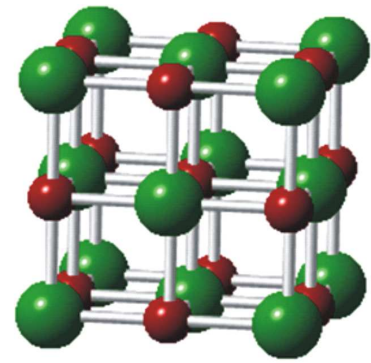

(a)

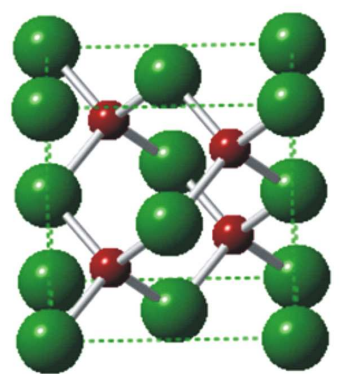

(c)

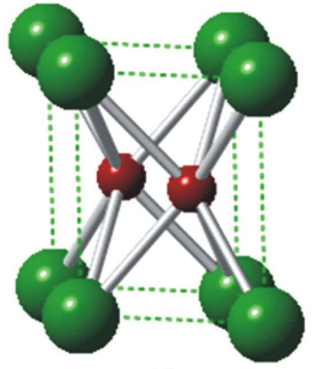

(e)

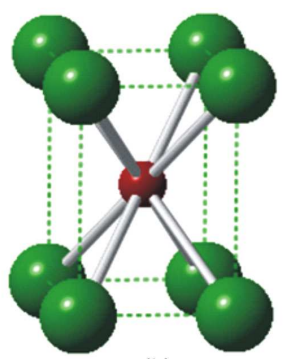

(b)

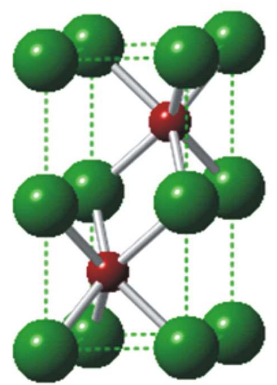

(d)

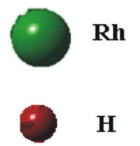

Fig. 1. The primitive unit cells of rhodium hydride: (a) NaCl-RhH, (b) CsCl-RhH, (c) ZB-RhH, (d) WCRhH, (e) NiAs-RhH.

The heat of formation of metal hydride is calculated by subtracting the total energies of the host alloy and the absorbed hydrogen molecule from that of the final state

$$
\Delta H=E_{\mathrm{MH}}-E_{\mathrm{M}}^{a}-E_{\mathrm{H}}^{a},
$$

where $E_{\mathrm{M}}^{a}$ and $E_{\mathrm{H}}^{a}$ are the energies of an isolated metal and hydrogen atom, respectively, $E_{\mathrm{MH}}$ is the energy of the metal hydride. The stability of a hydride depends on 
its formation energy through the reaction

$$
x y \mathrm{M}+\mathrm{H}_{2} \rightarrow x \mathrm{M}_{y} \mathrm{H}_{2 / x} .
$$

The formation energy is a useful parameter in the determination of relative stability. The calculated formation energies for different structure are given in Table I. It is found that the formation energy of $\mathrm{RhH}$ in $\mathrm{NaCl}$ structure is minimal, when compared to other structures. So $\mathrm{RhH}$ is more stable in $\mathrm{NaCl}$ structure. This may be due to hybridization between the Rh $d$ state and the hydrogen $s$ state.

\subsection{Mechanical stability of rhodium hydride}

The stability of crystal structures can be evaluated through elastic constants. Elastic constants are the measure of the resistance of a crystal to an externally applied stress. In order to calculate the elastic constants of a structure, a small strain is applied onto the structure and its stress is determined. The energy of a strained system $[44,45]$ can be expressed in terms of the elastic constants $C_{i j}$ as

$$
\begin{gathered}
\frac{\Delta E}{V_{0}}=\frac{E\left(\left\{e_{i}\right\}\right)-E_{0}}{V_{0}}=\left(1-\frac{V}{V_{0}}\right) P\left(V_{0}\right) \\
+\frac{1}{2}\left(\sum_{1}^{6} \sum_{1}^{6} C_{i j} e_{i} e_{j}\right)+\mathrm{O}\left(\left\{e_{i}^{3}\right\}\right),
\end{gathered}
$$

where $V_{0}$ is the volume of the unstrained lattice, $E_{0}$ is the total minimum energy at this unstrained volume of the crystal, $P\left(V_{0}\right)$ is the pressure of the unstrained lattice, and $V$ is the new volume of the lattice due to strain tensor [45]. The elasticity tensor has three independent components $\left(C_{11}, C_{12}, C_{44}\right)$ for cubic crystals and five $\left(C_{11}, C_{12}, C_{44}, C_{13}, C_{33}\right)$ for hexagonal crystals. A proper choice of the set of strains $\left\{e_{i}, i=1,2, \ldots, 6\right\}$, in Eq. (3) leads to a parabolic relationship between $\Delta E / V_{0}\left(\Delta E \equiv E-E_{0}\right)$ and the chosen strain [46]. For each lattice structure of $\mathrm{RhH}$, the lattice is strained by $0 \%, \pm 1 \%$, and $\pm 2 \%$ to obtain their total minimum energies $E(V)$. These energies and strains are fitted with the corresponding parabolic equations of $\Delta E / V_{0}$ to yield the required second-order elastic constants [46]. While computing these energies, all atoms are allowed to relax with the cell shape and volume fixed by the choice of strains $\left\{e_{i}\right\}$. From the calculated $C_{i j}$ values, the bulk modulus and shear modulus for the cubic crystals and hexagonal crystals are determined using the VoigtReuss-Hill (VRH) averaging scheme [47-49]. The strain energy $(1 / 2) C_{i j} e_{i} e_{j}$ of a given crystal in Eq. (3) must always be positive for all possible values of the set $\left\{e_{i}\right\}$; for the crystal to be mechanically stable. For a stable hexagonal structure, the five independent elastic constants $C_{i j}\left(C_{11}, C_{12}, C_{33}, C_{13}, C_{44}\right)$ should satisfy the well known Born-Huang criteria [50], while for a cubic crystal, the three independent elastic constants $C_{i j}\left(C_{11}\right.$, $C_{12}, C_{44}$ ) should satisfy the Born-Huang criteria [50].

The calculated elastic constants $C_{i j}(\mathrm{GPa})$, the Young's modulus $E$ (GPa) [51], shear modulus $G(\mathrm{GPa})$ [51] and Poisson's ratio $(\nu)$ [52] are given in Table II.
TABLE II

Calculated elastic constants $C_{11}, C_{12}, C_{44}, C_{13}, C_{33}$ (GPa), Young's modulus $E(\mathrm{GPa})$, shear modulus $G$ (GPa), and Poisson's ratio $\nu$.

\begin{tabular}{c|c|c|c|c|c}
\hline \hline & RS-RhH & ZB-RhH & CsCl-RhH & Wurtzite-RhH & NiAs-RhH \\
\hline$C_{11}$ & 384 & 399 & 374 & 343 & 434 \\
$C_{12}$ & 109 & 127 & 154 & 92 & 138 \\
$C_{44}$ & 102 & 112 & 127 & 57 & 60 \\
$C_{13}$ & - & - & - & 55 & 58 \\
$C_{33}$ & - & - & - & 347 & 350 \\
$E$ & 290 & 308 & 304 & 299 & 337 \\
$G$ & 116 & 122 & 119 & 126 & 140 \\
$\nu$ & 0.22 & 0.24 & 0.29 & 0.19 & 0.21
\end{tabular}

Clearly, the computed elastic constants for both cubic and hexagonal RhH satisfy Born-Huang criteria, suggesting that they are mechanically stable. Young's modulus $(E)$ and Poisson's ratio $(\nu)$ are the two important factors for technological and engineering application. The stiffness of the solid can be analyzed using the Young modulus $(E)$ value. The larger the value of $E$, the stiffer is the material. From Table II, it is observed that among the five phases cubic $\mathrm{ZB}-\mathrm{RhH}$ is the stiffest phase. Among the considered phases, the Poisson ratio of rocksalt-RhH is lower than that of $\mathrm{ZB}, \mathrm{CsCl}$, wurtzite and NiAs phases, indicating that the $\mathrm{Rh}-\mathrm{H}$ bonding is more directional in the rock salt phase.

\subsection{Structural phase transition}

At ambient condition, $\mathrm{RhH}$ is stable with $\mathrm{NaCl}$ structure. Under high pressure it undergoes a structural phase transition. The total energies are plotted as a function of reduced volume in Fig. 2 for all the five possible structures. From Fig. 2, it is seen that $\mathrm{RhH}$ is stable with $\mathrm{NaCl}$ structure up to $11 \mathrm{GPa}$, and it has undergone a structural phase transition under high pressure. In order to calculate the transition pressure the Gibbs free energy is calculated for the two phases using the expression

$$
G=E_{\text {tot }}+P V-T S \text {. }
$$

Since the theoretical calculations are performed at $0 \mathrm{~K}$, the Gibbs free energy will become equal to the enthalpy $(H)$. Hence,

$$
H=E_{\text {tot }}+P V \text {. }
$$

At a given pressure, a stable structure is one in which the enthalpy has its lowest value. The transition pressures are calculated at which the enthalpies of the two phases are equal.

The enthalpy as a function of pressure is shown in Fig. 3. The phase transitions from $\mathrm{NaCl}$ to $\mathrm{ZB}$ occuring at $11 \mathrm{GPa}$ are shown in Fig. 3. On further increase in pressure to around $154 \mathrm{GPa}, \mathrm{ZB}-\mathrm{RhH}$ is transformed to $\mathrm{CsCl}-\mathrm{RhH}$. As the pressure is further increased to $382 \mathrm{GPa}$, we observe $\mathrm{CsCl}$ to $\mathrm{NiAs}$ phase transition. Thus, a series of $\mathrm{NaCl} \rightarrow \mathrm{ZB} \rightarrow \mathrm{CsCl} \rightarrow \mathrm{NiAs}$ structural phase transitions are observed in $\mathrm{RhH}$.

\subsection{Electronic structure}

The electronic structure of $\mathrm{Rh}$ and $\mathrm{RhH}$ with four possible structures is examined by looking at its density of 


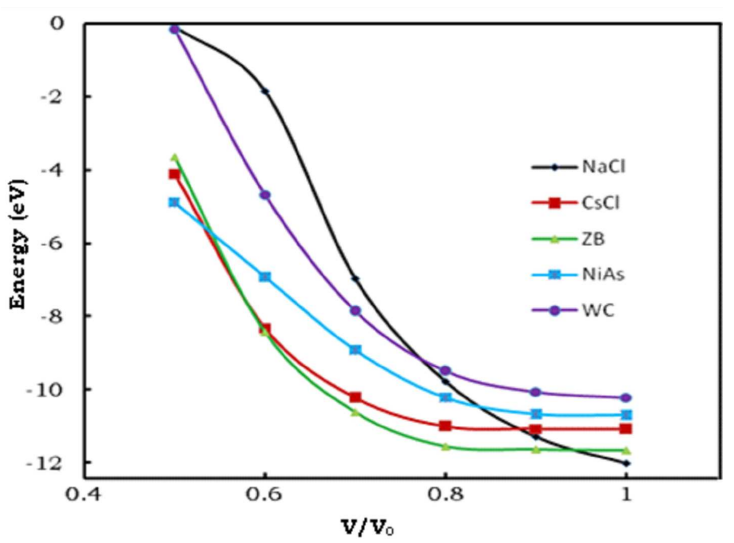

Fig. 2. The total energies as a function of volume.

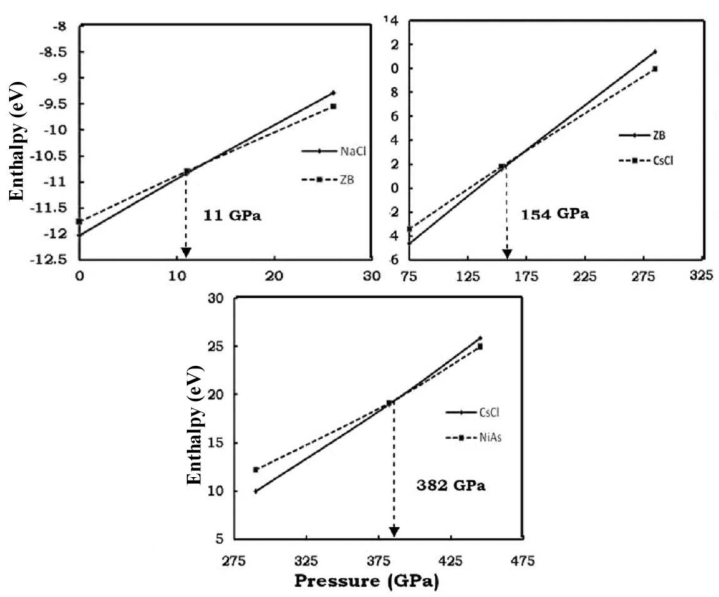

Fig. 3. The enthalpy as a function of pressure.

states (DOS). The total and partial DOS of rhodium and rhodium hydride is shown in Fig. 4 and Fig. 5. The analysis of DOS of rhodium and rhodium hydride shows some considerable changes in the valence band which is due to the addition of hydrogen atom. The DOS of both Rh and RhH exhibits metallic character at ambient pressure. The electronic structure is mostly dominated by the $d$-state of Rh atoms. Generally, it is known that the valence band is formed mainly due to the $\mathrm{Rh} d$ state electrons below the Fermi energy $\left(E_{\mathrm{F}}\right)$, while other states of $\mathrm{Rh}$ are broader and mainly centered above the Fermi energy within the conduction band. In the valence band of $\mathrm{RhH}$ the highest spikes are due to Rh $d$ state electrons and $\mathrm{H} s$ state electron. The range between -3 to $6 \mathrm{eV}$ is due to $s$ state electron of hydrogen atom and the range between 6 to $15 \mathrm{eV}$ is due to $d$ state electrons of rhodium atom. From Fig. 4 it is observed that the Fermi level of $\mathrm{RhH}$ is shifted upwards from pure Rh. Therefore it is concluded that the width of the valence state in $R h$ is increased due to the addition of hydrogen atom.

To further investigate the metallic properties of $\mathrm{RhH}$, the partial density of states (PDOS) is shown in Fig. 5. For $\mathrm{NaCl}$ structure, a deep valley called the pseudogap is

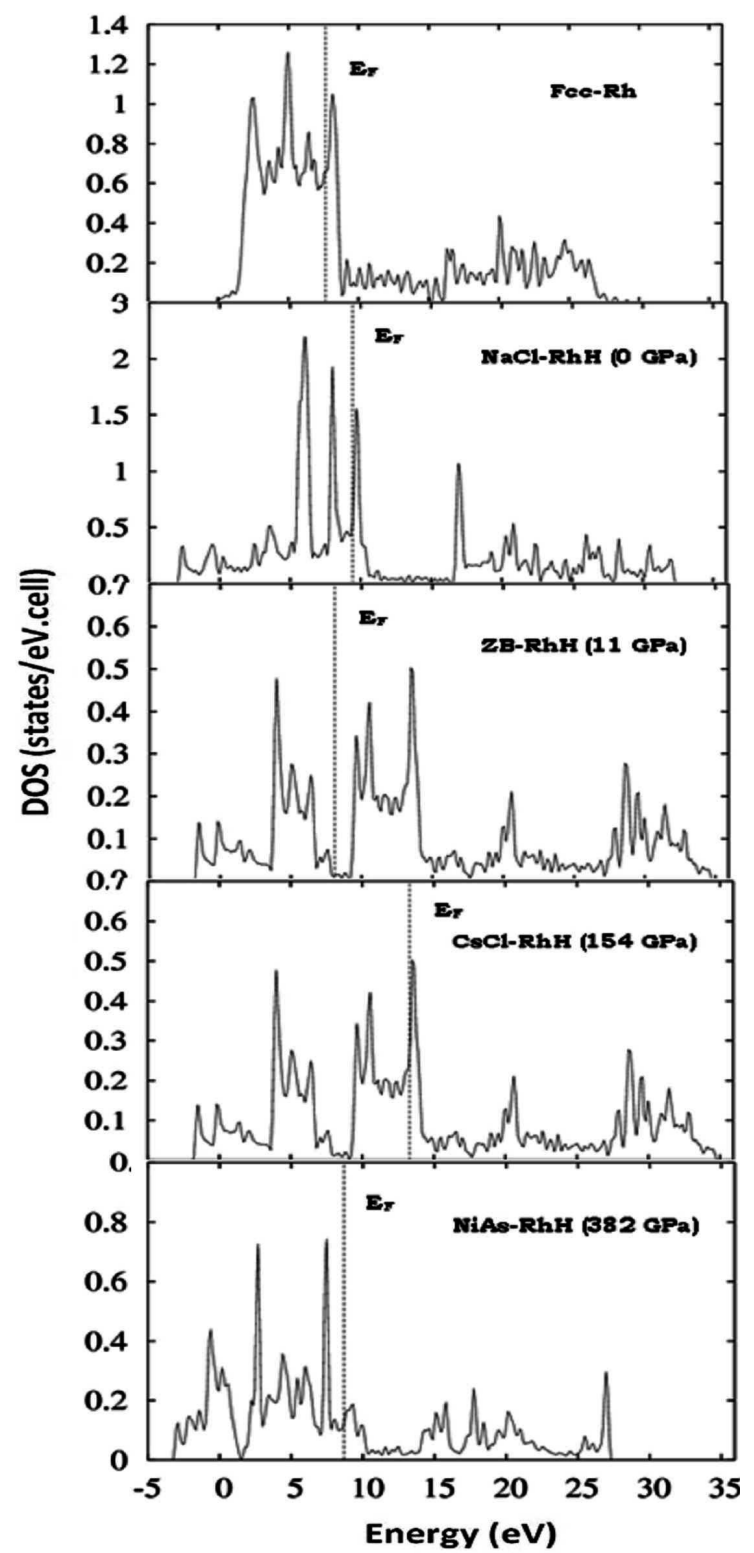

Fig. 4. Density of states of Rh and RhH.

found near the Fermi level, which results from the strong hybridization between Rh $d$ states and hydrogen $(\mathrm{H}) 1 s$ state and also indicate the significant covalent bonding. For the ZB structure, it is found that the energy region for the hybridization between $\mathrm{Rh} d$ states and hydrogen (H) $1 s$ state is from $-5 \mathrm{eV}$ to $15 \mathrm{eV}$. The main peak appearing at the energy region $10 \mathrm{eV}$ to $15 \mathrm{eV}$ is due to $\mathrm{Rh} d$ state electrons in $\mathrm{CsCl}$ structure. For NiAs structure, the peaks from $-5 \mathrm{eV}$ to $10 \mathrm{eV}$ are mainly due to the $d$ states of rhodium atom and $1 s$ state of hydrogen atom that also exhibit a strong hybridization.

\subsection{Clean rhodium surface and surface stability}

The surface energy is the energy required to create a surface from the bulk material. The relative stability 


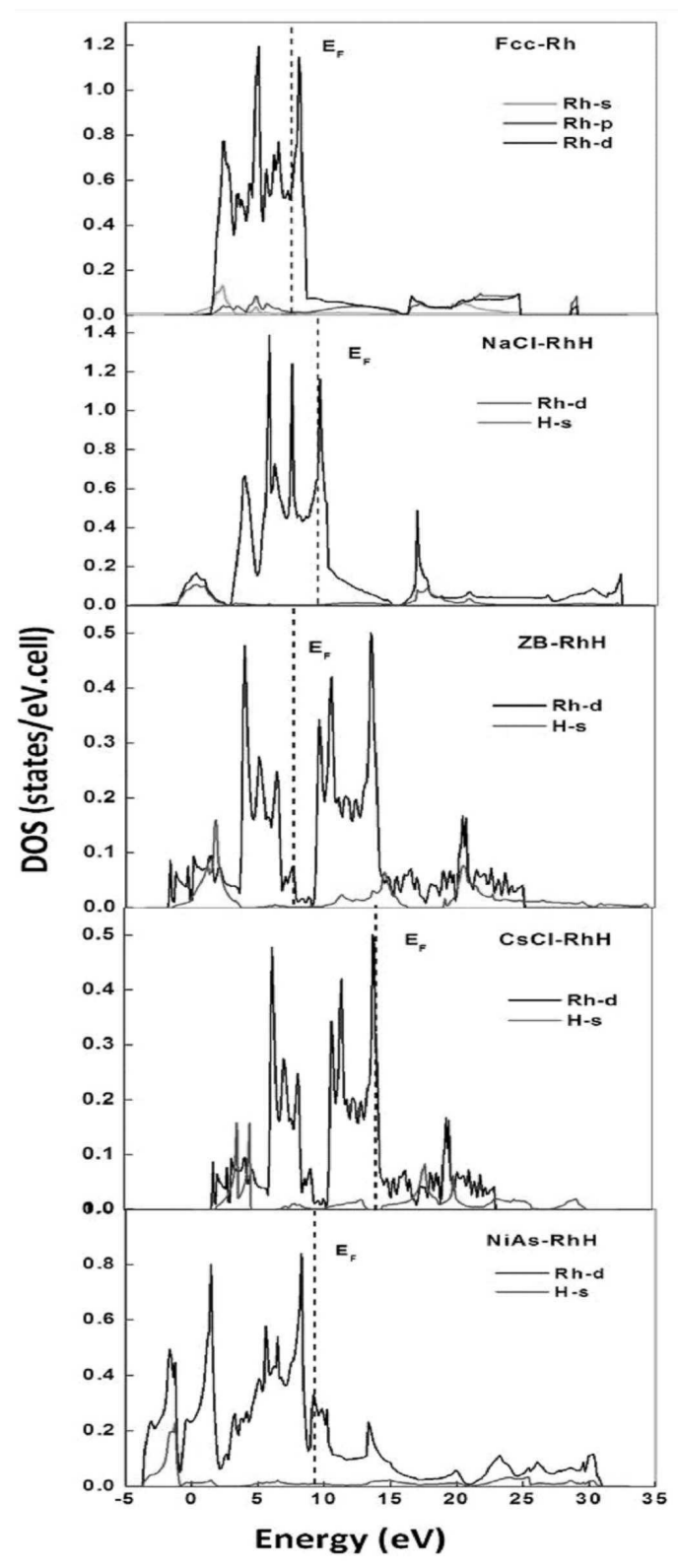

Fig. 5. Partial density of states of Rh and RhH.

of surfaces is estimated according to their surface energy $(\sigma)$, which can be expressed as

$$
\sigma=E_{\text {slab }}-N E_{\text {bulk }},
$$

where $E_{\text {slab }}, E_{\text {bulk }}$ is the total energy of the two-dimensional slab and the total energy of bulk rhodium, respectively. Here, we have analysed the stability of Rh (100) and Rh (111) surfaces for rhodium. The calculated surface energy for Rh (100) surface is $1.06349 \mathrm{eV}$ and for Rh (111) surface is $0.7539 \mathrm{eV}$. The clean Rh (100) surface calculation predicts inward relaxation of $-3.4 \%$ which is good agreement with the other theoretical results [53-58]. For Rh (111) surface, the predicted inward surface relaxation is $-1.09 \%$. It is found that the outward relaxation of the top layer is increased to $3.7 \%$ due to the adsorption of a monolayer of hydrogen. From the results one can see that the $\mathrm{Rh}$ (111) surface has a lower surface energy, and this should be a stable surface. Experimental results also showed that the (111) planes are the preferred cleavage planes for fcc $\operatorname{Rh}[59,60]$. We have computed the hydrogen adsorption energy and binding energy for the stable Rh (111) surface.

\subsection{Hydrogen adsorption on thin Rh (111) surface}

To determine the stable adsorption geometry we have allowed, in addition to the relaxation of the substrate, a minimization of the total energy with respect to the height of the adsorbed hydrogen atom measured from the centre of the top layer of the substrate. We considered only the top layer of the substrate among three different high-symmetry positions of the adsorbate (on top of a substrate atom, in a position forming a bridge between two nearest-neighbour surface atoms, and in fourfold hollows in the (111) surface of the face-centered cubic crystal). To determine the population of rhodium adatoms on the surface $N_{\mathrm{Rh}}$ we can define total hydrogen coverage $\Theta_{\text {tot }}$ as the ratio of the total uptake of $\mathrm{H}$ adatoms $N_{\mathrm{H}}$ to the number of Rh atoms on the surface

$$
\Theta_{\text {tot }}=N_{\mathrm{H}} / N_{\mathrm{Rh}} \text {. }
$$

We have investigated the topmost layer of rhodium at which the hollow site is occupied by hydrogen atoms. In order to explore the adsorption of hydrogen at high coverage, guided by experiment [56], four phases for hydrogen adsorption at the most favored site have been considered. The four phases $3 \times 1,4 \times 1,5 \times 1$, and $6 \times 1$ correspond to the coverage of $0.333,0.25,0.2$, and $0.166 \mathrm{ML}$, respectively. The adsorption energy $E_{\text {ads }}$ is defined as the energy difference between the hydrogen-covered surface and the clean surface plus $N_{\mathrm{H}} / 2$ hydrogen molecules

$$
E_{\mathrm{ads}}=-\frac{1}{N_{\mathrm{H}}}\left[E_{\mathrm{M}: \mathrm{H}}(\Theta)-E_{\mathrm{M}}-\frac{N_{\mathrm{H}}}{2} E_{\mathrm{H}_{2}}\right],
$$

where $E_{\mathrm{H}-\mathrm{Rh}}$ and $E_{\mathrm{Rh}}$ are the total energy of the $\mathrm{Rh}(111)$ system with and without $\mathrm{H}$ atoms adsorbed on the surface, $N_{\mathrm{H}}$ denotes the number of $\mathrm{H}$ atoms. $E_{\mathrm{H} 2}$ represents the energy of the isolated $\mathrm{H}$ atom.

The binding energy as a function of the coverage $\Theta$ is defined as the difference between the total energies of the hydrogen-covered surface $E_{\mathrm{M}: \mathrm{H}}$ and the clean surface $E_{\mathrm{H}}$ plus the total energy of the appropriate number of hydrogen atoms. The binding energy can be expressed as

$$
E_{\mathrm{b}}=-\frac{1}{N_{\mathrm{H}}}\left[E_{\mathrm{M}: \mathrm{H}}(\Theta)-E_{\mathrm{M}}-N_{\mathrm{H}} E_{\mathrm{H}}\right] .
$$

The adsorption energies and structural properties for hydrogen atoms adsorption at different coverages and binding energies are presented in Table III. From Table III, it is seen that adsorption energy at the coverage of 0.333 is found to be about $1.8518 \mathrm{eV}$. As the coverage is increased, the adsorption energy is found to be slightly increased, which indicates that the hydrogen adsorption energy depends on coverage.

From the calculated structural properties of hydrogen adsorption on the $\mathrm{Rh}(111)$ surface, the vertical distance 


\section{TABLE III}

Adsorption energies, the changes of the interlayer distances $\left(\Delta d_{i j}\right)$ as the percentages of the distance in the bulk and structural parameters for the adsorption of hydrogen at hollow sites during the coverage range from 0.333 to $0.166 \mathrm{ML}$.

\begin{tabular}{c|c|c|c|c|c}
\hline \hline Phase & $\begin{array}{c}\text { Adsorption } \\
\text { energy } \\
E_{\text {ads }}[\mathrm{eV}]\end{array}$ & $\begin{array}{c}\text { Binding } \\
\text { energy }\end{array}$ & $\begin{array}{c}\Delta d_{12} / d_{0}[\mathrm{eV}] \\
{[\%]}\end{array}$ & $\begin{array}{c}\Delta d_{23} / d_{0} \\
{[\%]}\end{array}$ & $\begin{array}{c}h \\
{[\AA]}\end{array}$ \\
\hline $3 \times 1$ & 1.8518 & 4.0054 & 0.09 & 3.7 & 2.37 \\
$4 \times 1$ & 2.2325 & 4.7667 & -1.12 & 2.6 & 2.12 \\
$5 \times 1$ & 2.5351 & 5.4719 & -3.6 & 1.51 & 1.58 \\
$6 \times 1$ & 2.8617 & 6.0252 & -0.09 & 1.2 & 1.01
\end{tabular}

(h) between the hydrogen atom and the first Rh substrate layer for hollow site is determined to be 2.373 and $1.0057 \AA$ corresponding to the coverage of 0.33 and $0.16 \mathrm{ML}$, respectively.

The reason could be that the $\mathrm{H}-\mathrm{H}$ repulsion at high coverage is something effective in our calculation, and the similar result also has been found for the adsorption of $\mathrm{H}$ on Mo (110) surface [61]. It is found that the vertical distance $(h)$ between the hydrogen atom and the first $\mathrm{Rh}$ substrate layer is 2.373, 2.1259, 1.5844 and $1.057 \AA$ for the coverage of $0.333,0.25,0.2$ and 0.166 , respectively. It indicates that the hydrogen atoms move closely to the top layer $\mathrm{Rh}$ as the coverage is decreased.

Also from Table III, it is seen that, as the number of layers increases the binding energy increases. From the structural property of $\Delta d_{12} / d_{0}$ and $\Delta d_{23} / d_{0}$, we found an expansion of first interlayer spacing compared to clean $\mathrm{Rh}(111)$ surface while the second interlayer spacing is found to be in little contraction. It indicates that the atoms in the 1st layer move away from the second Rh layer while the atoms in the 2nd layer move close to the third Rh layer as the coverage increases. It is found that the expansion and contraction depend on the coverage. Our results show that adsorption of a monolayer of hydrogen strongly reduces the inward relaxation as the layer increases. Hence, it is found that the hydrogen-induced surface relaxation is strongly coverage dependent.

\section{Conclusions}

The electronic structure, high pressure phase transition and mechanical stability of rhodium hydride have been investigated using ab initio calculations. The predicted new high pressure phase of rhodium hydride is hexagonal NiAs type. The density-functional theory with generalized gradient approximation has been used to study surface stability and hydrogen adsorption on Rh (111) surface. Our results suggest that the Rh (111) surface is more stable than the Rh (100) surface. The adsorption energy and binding energy increases with increase in the number of layers. The maximum adsorption energy is achieved in $6 \times 1$ phase with the value of $2.8617 \mathrm{eV}$. Hence, the adsorption of hydrogen may be very strong in $6 \times 1$ phase.

\section{Acknowledgments}

We thank our college management for their constant encouragement. The financial assistance from UGC (MRP.F.No.38-141/2009), India is duly acknowledged with thanks.

\section{References}

[1] Z.A. Schlapbach, Nature 414, 353 (2001).

[2] A.V. Irodova, V.P. Glazkov, V.A. Somenkov, S.Sh. Shilshtein, V.E. Antonov, E.G. Ponyatovskii, Sov. Phys. Crystallogr. 33, 453 (1988).

[3] Xin Cui, Jian-Tao Wang, Xi-Xia Liang, Guo-Zhong Zhao, Solid State Commun. 149, 322 (2009).

[4] V.V. Bannikov, I.R. Shein, A.L. Ivanovski, J. Struct. Chem. 51, 956 (2010).

[5] Xin Cui, Jian-Tao Wang, Xi-Xia Liang, Guo-Zhong Zhao, Chin. Phys. Lett. 27, 027101 (2010).

[6] G.C. Bond, Catalysis by Metals, Academic Press, London 1962, p. 320.

[7] O. Beeck, Discuss. Faraday Soc. 8, 118 (1950).

[8] C. Kemball, Proc. R. Soc. Lond. A 214, 413 (1952).

[9] J.R. Anderson, C. Kemball, Proc. R. Soc. Lond. A 223, 361 (1954).

[10] J.T. Yates Jr., P.A. Thiel, W.H. Weinberg, Surf. Sci. 82, 45 (1979).

[11] G.A. Mills, F.W. Steffgen, Catal. Rev. 8, 159 (1974).

[12] V.J. Mimeault, R.S. Hansen, J. Chem. Phys. 45, 2240 (1966).

[13] M.G. Wells, N.W. Cant, R.G. Greenler, Surf. Sci. 67, 541 (1979).

[14] S.M. Edwards, R.P.H. Gasser, D.P. Green, D.S. Hawkins, A.J. Stevens, Surf. Sci. 72, 213 (1978).

[15] D.G. Castner, B.A. Sexton, G.A. Somorjai, Surf. Sci. 71, 519 (1978).

[16] K. Christmann, M. Ehsasi, W. Hirschwald, J.H. Block, Chem. Phys. Lett. 131, 192 (1986).

[17] H. Conrad, W. Stenzel, M.E. Kordesch, in: Fifth Int. Conf. on Vibration at Surfaces, Grainnau (Germany), Eds. A.M. Bradshaw, H. Conrad, Elsevier, Amsterdam 1987, p. 1.

[18] W. Nichtl, N. Bickel, L. Hammer, K. Heinz, K. Muller, Surf. Sci. 188, L729 (1987).

[19] E. Ehsasi, K. Christmann, Surf. Sci. 194, 172 (1988).

[20] W. Oed, W. Puchta, N. Bickel, K. Heinz, W. Nichtl, K. Muller, J. Phys. C, Solid State Phys. 21, 237 (1988).

[21] K. Christmann, Mol. Phys. 66, 1 (1989).

[22] J.T. Yates Jr., P.A. Thiel, W.H. Weinberg, Surf. Sci. 84, 427 (1979).

[23] A. Eichler, J. Hafner, G. Kresse, J. Condens. Matter 8, 7659 (1996).

[24] R. Atta-Fynn, A.K. Ray, Physica B 400, 307 (2007).

[25] M. Evecen, M. Cakmak, Physica B 405, 4059 (2010).

[26] G. Kresse, J. Hafner, Phys. Rev. B 47, 558 (1993); ibid. 49, 14251 (1994). 
[27] G. Kresse, J. Furthmuller, Comput. Mater. Sci. 6, 15 (1996).

[28] G. Kresse, J. Furthmuller, Phys. Rev. B 54, 11169 (1996).

[29] P.E. Blochl, Phys. Rev. B 50, 17953 (1994).

[30] G. Kresse, D. Joubert, Phys. Rev. B 59, 1758 (1999).

[31] J.P. Perdew, J.A. Chevary, S.H. Vosko, K.A. Jackson, M.R. Pederson, D.J. Singh, C. Fiolhais, Phys. Rev. B 46, 6671 (1992).

[32] H.J. Monkhorst, J.D. Pack, Phys. Rev. B 13, 5188 (1976).

[33] H. Smithson, C.A. Marianetti, D. Morgan, A. Van der Ven, A. Predith, G. Ceder, Phys. Rev. B 66, 144107 (2002).

[34] F.R. de Boer, R. Boom, W.C.M. Matten, A.R. Miedema, A.K. Niessen, in: Cohesion in Metals: Transition Metal Alloys, Eds. F.R. de Boer, D.G. Pettifor, Vol. 1, North-Holland, Amsterdam 1988.

[35] J.E. Shirber, B. Morosin, Phys. Rev. B 12, 117 (1975).

[36] J.E. Worsham, M.K. Wilkinson, C.G. Shull, J. Phys. Chem. Solids 3, 303 (1957).

[37] S. Kanagabrabha, M. Santhosh, G. Sudhapriyanga, A.T. Asvinimeenaatci, R. Rajeswarapalanichamy, K. Iyakutti, AIP Conf. Proc. 1536, 919 (2013).

[38] S. Kanagabrabha, R. Rajeswarapalanichamy, K. Iyakutti, Int. J. Metals 2013, 743652 (2013).

[39] S. Kanagabrabha, A.T. Asvinimeenaatci, G. Sudhapriyanga, A. JemmyCinthia, R. Rajeswarapalanichamy, K. Iyakutti, Acta Phys. Pol. A 123, 126 (2013).

[40] A. Machida, Phys. Rev. Lett. 108, 205501 (2012).

[41] S.Sh. Shilstein, V.P. Glazkov, A.V. Irodova, V.A. Somenkov, V.E. Antonov, E.G. Ponyatovskii, Z. Phys. Chem. N.F. 146, 129 (1985).

[42] F.D. Murnaghan, Proc. Natl. Acad. Sci. USA 30, 244 (1944).
[43] H. Gou, L. Hou, J. Zhang, F. Gao, Appl. Phys. Lett. 92, 241901 (2008).

[44] J.F. Nye, Physical Properties of Crystals, Oxford University Press, Oxford 1985.

[45] S.Q. Wu, Z.F. Hou, Z.Z. Zhu, Solid State Commun 143, 425 (2007).

[46] M. Kalay, H.H. Kart, T. Cagin, J. Alloys Comp. 484, 431 (2009).

[47] W. Voigt, Handbook of Crystal Physics, Terubner, Leipzig 1928 (in German).

[48] A. Reuss, Z. Angew. Math. Mech. 9, 49 (1929).

[49] R. Hill, Proc. Phys. Soc., Lond. A 65, 349 (1952).

[50] M. Born, K. Huang, Dynamical Theory of Crystal Lattices, Clarendon, Oxford 1956.

[51] K.B. Panda, K.S.R. Chandran, Acta Mater. 54, 1641 (2006).

[52] P. Ravindran, L. Fast, P.A. Korzhavyi, B. Johansson, J. Wills, O. Eriksson, J. Appl. Phys. 84, 4891 (1998).

[53] D.R. Hamann, P.J. Feibelman, Phys. Rev. B 37, 3847 (1988).

[54] P.J. Feibelman, D.R. Hamann, Surf. Sci. 234, 377 (1990).

[55] P.J. Feibelman, Phys. Rev. Lett. 67, 461 (1991).

[56] P.J. Feibelman, Phys. Rev. B 43, 9452 (1991).

[57] M. Methfessel, D. Hennig, M. Scheffler, Phys. Rev. B 46, 4816 (1992).

[58] A. Eichler, J. Hafner, J. Furthmuller, G. Kresse, Surf Sci. 346, 300 (1996).

[59] J.T. Yates, P.A. Theil, W.H. Weinberg, Surf. Sci. 84, 427 (1979).

[60] J.T. Yates, P.A. Theil, W.H. Weinberg, Surf. Sci. 82, 45 (1979).

[61] P. Jena, J. Phys. Chem. Lett. 2, 206 (2011). 